\title{
Neoclassical bootstrap current in solar plasma loops
}

\author{
B.-L. $\operatorname{Tan}^{1,2}$ and G.-L. Huang ${ }^{1}$ \\ 1 Purple Mountain Observatory, the Chinese Academy of Sciences, Nanjing, 210008, PR China \\ e-mail: bltan@pmo.ac.cn \\ 2 Graduate School of the Chinese Academy of Sciences, Beijing, 100871, PR China
}

Received 16 August 2005 / Accepted 26 February 2006

\begin{abstract}
From the similarities in magnetic configurations and plasma behaviors between tokamaks and solar current-carrying plasma loops, we apply the theory of neoclassical bootstrap current in tokamaks to the solar plasma loops. We present a simplified expression of the bootstrap current in the solar plasma loops and find that there may be a considerable component of the neoclassical bootstrap current in some compact current-carrying solar flare loops; e.g. the fraction of the bootstrap current is up to $44.6 \%$ of a flare loop of the event that occurred on Aug. 25, 1999. We suggest that the neoclassical effect changes the current distribution and affects the instability of solar plasma loops. Based on the data analysis of SXT/Yohkoh, HXT/Yohkoh, GOES, and NoRP, we find that the timescale of the neoclassical tearing modes is consistent with the rising time of the impulsive phase during the event, while the timescale of the classical tearing modes is much longer than that of the event, which may provide important evidence of the bootstrap current and help us understand the mechanisms of the eruptive events, such as solar flares, prominence, and CMEs.
\end{abstract}

Key words. Sun: evolution - plasmas - Sun: magnetic fields

\section{Introduction}

After many years of intensively studying of solar eruptive events (such as solar flares, prominence, and CMEs), there are still many unsolved problems for solar physics. The most essential questions include energy storage and release processes. About forty years ago, Alfvén \& Carlqvist (1967) stressed the importance of electric currents in the processes of solar flares. Recent observations show that current-carrying plasma loops may play an important role in the study of the solar eruptive events (Strong 1994; Spadaro 2003; Ashbourn \& Woods 2004). The technique of vector magnetograms proved the existence of the electric current and showed that the total current is about $\sim 10^{10}-10^{12} \mathrm{~A}$ (Severny 1964; Canfield et al. 1993). People think that such a current comes from the photospheric convective motion. Since we know that the loop current and its distribution dominate the plasma instabilities, evolution, energy storage, and release in solar plasma loops, studies of the origin and distribution of the current become a fundamental task (Sen \& White 1972; Zaitsev \& Stepanov 1992).

On the other hand, some theoretical studies from the early 1970s predicted that the effects of the finite toroidal orbit might lead to the generation of a new current, called the neoclassical bootstrap current, in the presence of the gradients of plasma density and temperature in toroidal plasma devices (Bickerton et al. 1971; Zarnstorff \& Prager 1986; Hirshman 1988; Peeters et al. 2000). Then, the experiments in many toroidal devices (in particular, tokamaks, toroidal multipoles, and stellarators) have confirmed the existence of the self-generated current (reviewed by Kikuchi \& Azumi 1995). Recently, a direct measurement of neoclassical bootstrap current in laboratory was made by Petty et al. (2005). By comparing magnetic configurations and plasma behaviors between tokamaks and solar current-carrying plasma loops, we suggest in this work that, besides the convective current, the bootstrap current may be an important component in solar current-carrying plasma loops.

In Sect. 2 we compare the magnetic configurations and plasma behavior in tokamaks and solar plasma loops. The derivations of bootstrap current in tokamaks and solar currentcarrying plasma loops are given in Sect. 3. A solar flare event is studied with analysis of bootstrap current in Sect. 4, and the conclusion and discussions are summarized in Sect. 5.

\section{Comparison between tokamaks and solar plasma loops}

There are a lot of similarities in the plasma and magnetic configurations between tokamaks and solar current-carrying plasma loops, which we presents in details.

\subsection{In tokamaks}

The magnetic field is composed of a toroidal component and a poloidal component in the tokamak. The toroidal component, $B_{\varphi}$ is the main part in the total magnetic field. The poloidal magnetic field, $B_{\theta}$ is induced by the plasma current flowing along the toroidal direction in the main coils, and $B_{\theta}$ depends on the distribution of the plasma current, $j_{\varphi}(r)$. From Fig. 1, we may clearly get insight of the configuration. Figure 1a shows that the magnetic lines stretch along the toroidal direction, rotate along the poloidal direction, and form a series of nested magnetic flux surfaces. Figure $1 \mathrm{~b}$ shows the spatial distribution of the magnetic field lines on a magnetic flux surface. Generally, $B_{\varphi} \gg B_{\theta}$, the total magnetic field can be approximately simplified to:

$\boldsymbol{B}=\boldsymbol{B}_{\varphi}+\boldsymbol{B}_{\theta} \simeq \frac{B_{0}}{1+(r / R) \cos \theta} \sim B_{0}(1-\epsilon \cos \theta)$. 


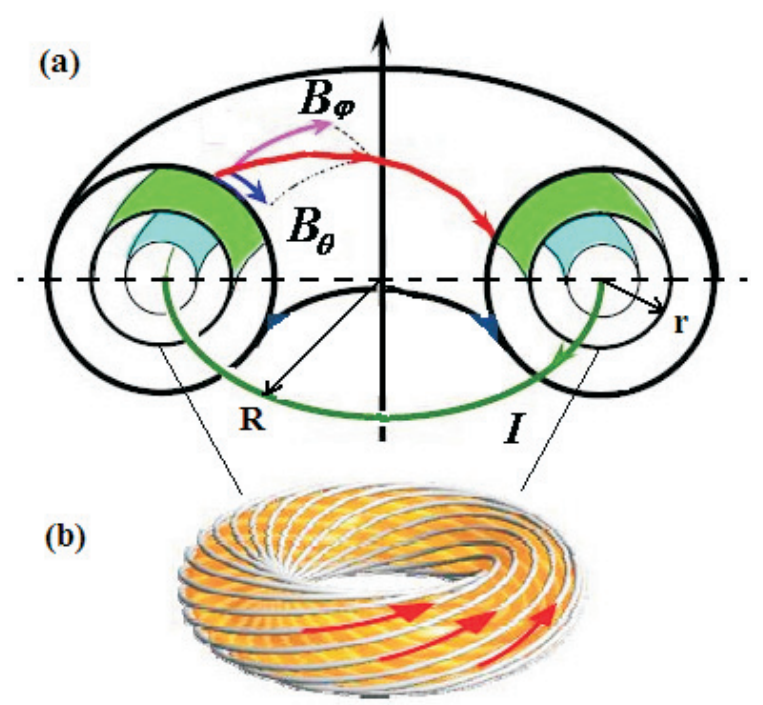

Fig. 1. Sketch maps of the magnetic configuration of the tokamak: a) the magnetic field lines form a series of nested magnetic flux surfaces; b) the spatial distribution of the magnetic field lines on a flux surface.

The maximum and minimum magnetic field strength on the magnetic flux surface are $B_{\max }=B_{0}(1+\epsilon)$ and $B_{\min }=B_{0}(1-\epsilon)$, respectively, here $B_{0}$ is the value on the toroidal axis of tokamaks, $r$ the cross-section radius of the flux surface, $R$ the main radius of tokamaks, and $\epsilon=r / R$ is the inverse aspect ratio of the flux surface. A magnetic line passes strong and weak magnetic field regions alternately, while it stretches in the toroidal direction and rotates in the poloidal direction in the tokamaks. The strong field region is near the inside part of the flux surface, while the weak field region is near the outside part of the flux surface. Thus a localized magnetic mirror forms, spreads on the magnetic surface, stretches in the toroidal direction, and rotates in the poloidal direction. The magnetic mirror ratio is: $\zeta_{t}=\frac{B_{\max }}{B_{\min }} \simeq \frac{1+\epsilon}{1-\epsilon}$.

Because of the existence of the localized magnetic mirror, the electrons in tokamaks may be classified into two groups: trapped and passing electrons. The trapped electrons cannot pass through the strong field region, so they only move and bounce back and forth along the banana orbit in the mirror. The magnetic mirror ratio $\zeta_{\mathrm{t}}$ is an important factor, which dominates the fraction of banana particles (i.e. trapped particles) relative to the total particles, and affects the magnitude of the bootstrap current.

Besides the configuration of localized magnetic mirror, the occurrence of the bootstrap current needs another two conditions: banana regime and pressure gradient. The banana regime depends on the plasma collisions. We may define a factor $g$ as a characteristic of the plasma collision, $g=\frac{\lambda_{\mathrm{c}}}{L} \sim 1.20 \times 10^{16} \frac{T_{\mathrm{e}}^{2}}{n_{\mathrm{e}} L}$, here $\lambda_{\mathrm{c}}$ is the electron mean free path, $L$ the scale length of the loops, $T_{\mathrm{e}}$ the electron temperature, the unit of $T_{\mathrm{e}}$ is $\mathrm{eV}$, and $n_{\mathrm{e}}$ the electron density with unit of $\mathrm{m}^{-3}$. When $g>1$, most of the trapped particles can finish one or more while bounces before de-trapping due to a scattering collision in the magnetic mirror; then the plasma situates in banana regime, and this condition is satisfied in most of modern tokamaks. And the gradient of the plasma pressure also exists in practice.

\subsection{In solar plasma loops}

The solar plasma loop is current-carrying in most cases (Ashbourn et al. 2004). Its magnetic field is also composed of

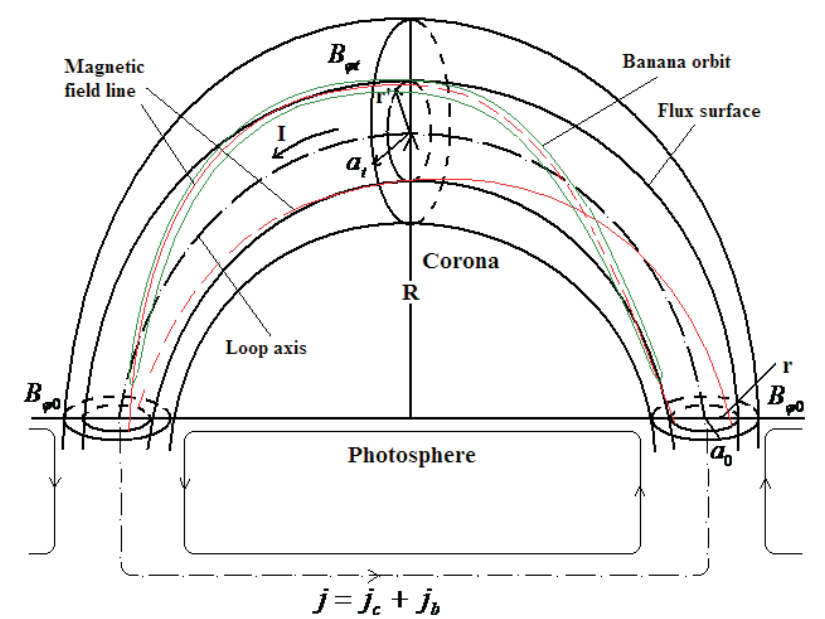

Fig. 2. Sketch maps of the magnetic configuration in the solar currentcarrying plasma loops and the banana orbit spread of the trapped particles (green lines), here red lines indicate the magnetic field lines.

a loop component and a poloidal component, which is similar to that of tokamaks. Figure 2 shows the magnetic configuration of solar current-carrying plasma loops. The poloidal component is induced by the electric current in the loops, while the current may come from the mass convection of the photosphere and subphotosphere. The magnetic field lines rotate around the axis of the loop, while they stretch along the loop, and also form a series of nested magnetic flux surfaces similar to those of tokamaks.

The loop component of the magnetic field in the solar plasma loop is changeable and different from the one in tokamaks. The magnetic field in foot-point is stronger than that in loop-top. The magnetic field $\left(B_{\varphi 0}\right)$ at foot-point can be obtained from the observation of vector magnetograms, and the one at loop-top can be extrapolated from certain models. The following is a simple extrapolated expression:

$B_{\varphi \mathrm{t}}=f B_{\varphi 0},(f<1)$,

where $B_{\varphi \mathrm{t}}$ is the loop magnetic field at the loop-top, $f$ a extrapolated function related with the loop parameters (such as height $h$, distance $d$ between the two foot-points of the loop, etc.). Thus, there are two strong magnetic field regions at two foot-points and a weak region at the loop-top, which forms a local magnetic mirror.

Let $a_{0}$ and $a_{\mathrm{t}}$ refer to the cross-section radii of the footpoints and the loop-top, respectively. Based on the conservation of magnetic flux along the loop $\left(a_{\mathrm{t}}^{2} B_{\varphi \mathrm{t}}=a_{0}^{2} B_{\varphi 0}\right)$, we have the relation:

$a_{\mathrm{t}}=f^{-1 / 2} a_{0}$.

On a flux surface with the cross-section radius $r$ at the footpoints and $r^{\prime}$ at the loop-top $\left(r^{\prime}=f^{-1 / 2} r\right)$, the poloidal magnetic field is estimated by

$B_{\theta \mathrm{f}}(r)=\frac{1}{c} \cdot \frac{2 I(r)}{r}=\frac{4 \pi}{c} \cdot \frac{1}{r} \int j(r) r \mathrm{~d} r$
$B_{\theta \mathrm{t}}\left(r^{\prime}\right)=\frac{1}{c} \cdot \frac{2 I\left(r^{\prime}\right)}{r^{\prime}}=\frac{4 \pi}{c} \cdot \frac{1}{r^{\prime}} \int j\left(r^{\prime}\right) r^{\prime} \mathrm{d} r^{\prime}$

Here $B_{\theta \mathrm{f}}(r)$ and $B_{\theta \mathrm{t}}\left(r^{\prime}\right)$ are the poloidal magnetic field at footpoints and loop-top, respectively, and $I(r)(j(r))$ and $I\left(r^{\prime}\right)\left(j\left(r^{\prime}\right)\right)$ are the total current (density) at foot-points and loop-top, respectively. The total current may be estimated from the solar observations of vector magnetograms (Canfield et al. 1993), which are 
obtained in the solar photosphere; thus, only the vertical electric current can be determined. However, with the assumption of a force-free field ( $\beta \ll 1$ and $\boldsymbol{j} \times \boldsymbol{B} \simeq 0$ ), we may estimate the magnitude of the total current from the vector magnetic field as: $j=\frac{B}{B_{z}} j_{z}, B=\left(B_{x}^{2}+B_{y}^{2}+B_{z}^{2}\right)^{1 / 2}$. The total current $I$ is obtained by integrating $j(r)$. Since the coronal loops are always in steady-state during a relatively long period, we may suppose that the distribution of total current is dominated by the pinch effect (Bennett 1934), with expressions such as:

$j(r)=\frac{2 I}{\pi} \frac{a_{0}^{2}}{\left(a_{0}^{2}+r^{2}\right)^{2}}=\frac{2 I}{\pi a_{0}^{2}} \frac{1}{\left(1+x^{2}\right)^{2}}$,

$j\left(r^{\prime}\right)=\frac{2 I}{\pi} \frac{a_{t}^{2}}{\left(a_{t}^{2}+r^{\prime 2}\right)^{2}}=\frac{2 I}{\pi a_{0}^{2}} \frac{f}{\left(1+x^{2}\right)^{2}}=f \cdot j(r)$,

where $x=r / a_{0}$, and $I$ refers to the total current in the loop. Substituting Eqs. (6) and (7) into Eqs. (4) and (5), we may get the poloidal magnetic field at foot-points and loop-top, respectively:

$B_{\theta \mathrm{f}}(r)=\frac{4 I r}{c\left(a_{0}^{2}+r^{2}\right)}=\frac{2 I}{c a_{0}} \cdot \frac{2 x}{1+x^{2}}=B_{\theta 0} \frac{2 x}{1+x^{2}}$

$B_{\theta \mathrm{t}}\left(r^{\prime}\right)=\frac{4 I r^{\prime}}{c\left(a_{\mathrm{t}}^{2}+r^{\prime 2}\right)}=\frac{2 I}{c a_{0}} \cdot f^{1 / 2} \frac{2 x}{1+x^{2}}=B_{\theta 0} f^{1 / 2} \frac{2 x}{1+x^{2}}$

with the relation:

$B_{\theta \mathrm{t}}\left(r^{\prime}\right)=f^{1 / 2} B_{\theta \mathrm{f}}(r)$

where $B_{\theta 0}=\frac{2 I}{c a_{0}}$ is the maximum of poloidal magnetic field strength at the foot-point. When $B_{\varphi 0} \gg B_{\theta 0}$ and $B_{\varphi \mathrm{t}} \gg B_{\theta \mathrm{t}}$, the total magnetic fields at the foot-points and the loop-top can be expressed respectively as follows:

$\boldsymbol{B}_{\text {foot }}=B_{\varphi 0} \boldsymbol{e}_{\varphi}+B_{\theta 0} \boldsymbol{e}_{\theta} \simeq B_{\varphi 0} \boldsymbol{e}_{\varphi}$,

$\boldsymbol{B}_{\text {top }}=f B_{\varphi 0} \boldsymbol{e}_{\varphi}+B_{\theta t} \boldsymbol{e}_{\theta} \simeq f B_{\varphi 0} \boldsymbol{e}_{\varphi}$.

Then the magnetic mirror ratio of solar current-carrying plasma loops can be written as:

$\zeta(x)=\frac{B_{\max }}{B_{\min }}=\frac{B_{\text {foot }}}{B_{\text {top }}} \simeq \frac{1}{f}$.

As a result, electrons in solar current-carrying plasma loops can also be classified into trapped and passing electrons due to the existence of a localized magnetic mirror, similar to those in tokamaks.

In fact, there are two possible kinds of magnetic traps in the coronal loops. The first case is considered in the above paragraphs, where the cross-section is changeable along the loop axis. The second case is connected with the loop-type which the cross-section does not change, and its high values of magnetic field on the internal (with respect to the loop curvature center) surface of current-carrying loops and the horizontal stratification of plasma will also create magnetic traps. This kind of trap is more similar to that of tokamaks, and it will probably play an important role in the neoclassical effect. In practice, the first case of traps is more prevalent in the solar atmosphere. So we limit our discussion of the first case of traps in this work. As for the second case of traps, its analysis is similar to the first case, so we do not present a detailed description.

We estimate the plasma collision based on the data from the book by Bray et al. (1991). The magnitude of the electron mean free path about $1.0 \times 10^{2}-1.0 \times 10^{8} \mathrm{~m}$, and factor $g$ is about $10^{-5}-10$. We see that some of the loops satisfy the condition of the banana regime. For example, when $T_{\mathrm{e}}=1 \mathrm{keV}$, $n_{\mathrm{e}}=10^{15} \mathrm{~m}^{-3}$, the electron mean free path is $1.2 \times 10^{7} \mathrm{~m}$. In this case, if the loop has the scale $L<1.2 \times 10^{7} \mathrm{~m}$, the condition $g>1$ is satisfied. Note that the plasma loops we considered here mainly refers to the flare loops. Observations show that the flare loops with the above parameters are possible. For example, many solar flare loops have a temperature up to $1 \mathrm{KeV}$ and higher (Tanaka 1982; Bray et al. 1991). Moreover, the plasma pressure gradient exists due to the pinch effect with distribution:

$p(r)=\frac{I^{2} a^{2}}{2 \pi\left(a^{2}+r^{2}\right)^{2} c^{2}}=\frac{I^{2}}{2 \pi a^{2} c^{2}} \cdot \frac{1}{\left(1+x^{2}\right)^{2}}$.

Note that $a$ is the radius of the cross-section with different values at the foot-points and the loop-top. Then, we get the pressure gradient as:

$\frac{\mathrm{d} p}{\mathrm{~d} r}=-\frac{2 I^{2}}{\pi a^{2} c^{2}} \cdot \frac{x}{\left(1+x^{2}\right)^{3}}$

However, the difference between tokamaks and solar plasma loops is obvious. In tokamaks, the banana orbit is generated from the magnetic field curvature and the inhomogeneity in the direction of main radius $R$, while the banana orbit of the solar plasma loop is generated mainly from the inhomogeneity at the loop direction of $\varphi$. On the other hand, Tokamaks are annular symmetrical systems, while the solar plasma loops seem to be a semicircular loop. We regard the solar plasma loop as composed of two parts. The first part is above the photosphere and includes loop-top and its vicinity, and is located in the corona with low plasma density and high temperature. The plasma is completely ionized, the electron mean free path has a large value, and it can be treated as non-collision. The magnetic mirror is mainly localized in this part. The second part is localized in the photosphere and in the low transition region with high plasma density and low temperature and is partially ionized, while the electron mean free path is very small. As the plasma in the second part is partially ionized, it is a good conductor for the electric current. The two parts constitute integral circuit where the electric current can flow from one part to the other. The idea that the current exists in narrow channels passing through the solar atmosphere and is closed in the photosphere was mentioned first by Alfvén \& Carlqvist (1967). This property is very important for the work of the neoclassical bootstrap current in the solar plasma loops.

\section{Derivation of the bootstrap current}

When conditions for the neoclassical bootstrap current are satisfied, trapped electrons move along the banana orbit and bounce back and forth in the mirrors, and electrons will have a net momentum when they finish a whole bounce. The net momentum will transfer to passing electrons while the trapped electrons collide with the passing electrons. Consequently, an electric current occurs as the bootstrap current, which is carried by passing electrons in the direction parallel to the magnetic field lines.

\subsection{In tokamaks}

In tokamaks, when the condition of the banana regime is satisfied, the critical incidence angle $\theta_{\mathrm{c}}$ can be identified from the expression $\sin \theta_{\mathrm{c}}=\left(1 / \zeta_{\mathrm{t}}\right)^{1 / 2}$. If the incidence angle $\theta>\theta_{\mathrm{c}}$, electrons cannot pass through the stronger magnetic region and 
bounce back to the weak region before they reach the stronger region. When $\theta<\theta_{\mathrm{c}}$, they become passing electrons, and we may get the critical parallel velocity of the passing electrons: $v_{\mathrm{p} 0}=v_{\mathrm{th}} \cdot \cos \theta_{\mathrm{c}}=v_{\mathrm{th}} \sqrt{\frac{2 \epsilon}{1+\epsilon}}=v_{\mathrm{th}} \cdot \sqrt{\frac{2}{1+\epsilon}} \cdot \sqrt{\epsilon} \sim \sqrt{\epsilon} \cdot v_{\mathrm{th}}$, where $v_{\text {th }}$ is the thermal velocity. In the case of a straight cylinder, $\epsilon \rightarrow 0, v_{\mathrm{p} 0} \rightarrow 0$; then all the particles become passing particles, and no magnetic mirror exists. The fraction of trapped electrons is about $\epsilon^{\frac{1}{2}}$. The canonical toroidal angular momentum of the trapped particles $P_{\varphi}$ is an invariant:

$P_{\varphi}=m R B_{\varphi} \frac{v_{p}}{B}-\frac{e}{c} A_{\varphi}$,

where $A_{\varphi}$ is the toroidal component of the vector potential, and $\mathrm{d} A_{\varphi} / \mathrm{d} r=R B_{\theta}, B_{\varphi} \approx B$. Then the width of trapped particle orbits is approximately $w_{\mathrm{b}} \approx \frac{m_{\mathrm{e}} c v_{\mathrm{p}}}{e B_{\theta}}$, where $m_{\mathrm{e}}$ is the electron mass. The net momentum generated by the density gradient in the banana orbit is:

$P_{0}=\left(m_{\mathrm{e}} \epsilon^{1 / 2} w_{\mathrm{b}} \frac{\mathrm{d} n_{\mathrm{e}}}{\mathrm{d} r}\right) \cdot\left(\epsilon^{1 / 2} v_{\text {th }}\right)=\frac{m_{\mathrm{e}}}{e} \frac{2 c \epsilon^{3 / 2}}{B_{\theta}} T_{\mathrm{e}} \frac{\mathrm{d} n_{\mathrm{e}}}{\mathrm{d} r}$.

Here the trapped electrons will collide not only with the passing electrons, but also with the ions. When they collide with the passing electrons, they diffuse in the direction of magnetic field lines, and the scattering over an angle $\theta_{c}$ in velocity space takes place with a typical efficiency of $\frac{1}{\left(\theta_{c}\right)^{2}}$. The trapped electrons are localized to a part $\sim \epsilon^{1 / 2}$ of velocity space. The characteristic time of such diffusion is about $t_{\mathrm{d}}=v_{\mathrm{eff}}^{-1}=\left(\epsilon^{1 / 2}\right)^{2} v_{\mathrm{ee}}^{-1}$. The momentum exchange in unit time between passing and trapped electrons is $\frac{v_{\mathrm{ee}}}{\epsilon} P_{0}$. In the meantime the collision between the passing electrons and the ions make an anti-directional drift velocity $v_{\mathrm{d}}$ to the passing electrons. The momentum change in unit time related to the anti-directional drift is $v_{\mathrm{ei}} n_{\mathrm{e}} m_{\mathrm{e}} v_{\mathrm{d}}$. Equating these two expressions for momentum exchange of the passing electrons, we get:

$v_{\mathrm{d}}=\frac{v_{\mathrm{ee}}}{v_{\mathrm{ei}}} \cdot \frac{2 c \epsilon^{1 / 2}}{e n_{\mathrm{e}} B_{\theta}} T_{\mathrm{e}} \frac{\mathrm{d} n_{\mathrm{e}}}{\mathrm{d} r}$.

This net momentum will result in a electric current as:

$j_{\mathrm{bt}}=-n_{\mathrm{e}} e v_{\mathrm{d}}=-\frac{v_{\mathrm{ee}}}{v_{\mathrm{ei}}} \frac{2 c \epsilon^{1 / 2}}{B_{\theta}} T_{\mathrm{e}} \frac{\mathrm{d} n_{\mathrm{e}}}{\mathrm{d} r}$.

Since $v_{\mathrm{ee}} \sim v_{\mathrm{ei}}$, the bootstrap current in tokamaks can be expressed approximately as

$j_{\mathrm{bt}}=-\frac{2 c \epsilon^{1 / 2}}{B_{\theta}} T_{\mathrm{e}} \frac{\mathrm{d} n_{\mathrm{e}}}{\mathrm{d} r}$

This expression (20) is consistent with the results of Bickerton (1971), Cordey (1988) etc., here $\mathrm{d} n_{\mathrm{e}} / \mathrm{d} r$ refers to the plasma density gradient. The unit of the temperature $T_{\mathrm{e}}$ is eV. From Table 1 we know that the bootstrap current is detected with a maximal value up to $0.85 \mathrm{MA}$, and the fraction $f_{\mathrm{bt}}$ has been up to $77 \%$ in several large tokamak devices since 1988 (Wesson 1997), here $f_{\mathrm{bt}}=I_{\mathrm{bt}} / I=s \epsilon^{1 / 2} \beta_{\mathrm{p}}, I$ is the total current, $s$ a constant around $1 / 3, \beta_{\mathrm{p}}=\frac{p}{B_{\theta}^{2} /(8 \pi)}$ the plasma poloidal $\beta$ value, and the plasma pressure $p \simeq 2 n_{\mathrm{e}} T_{\mathrm{e}}$.

The bootstrap current theory is widely accepted in the field of nuclear fusions. It is one of the most important bases for designing the next step of advanced tokamaks, e.g. ITER. In fact, the bootstrap current occurs not only in tokamaks, but also in stellarators (Peeters 2000) and toroidal multipoles (Zarnstorff \& Prager 1986).
Table 1. Experimental results of bootstrap current in tokamak devices.

\begin{tabular}{lccc}
\hline \hline Tokamak Devices & time & $I_{\mathrm{b}}(M A)$ & $f_{\mathrm{bt}}(\%)$ \\
\hline TFTR & 1988 & 0.52 & 58 \\
JET & 1989 & 0.85 & 28 \\
JT -60 & 1990 & 0.37 & 77 \\
JT $-60 U$ & 1992 & 0.85 & 58 \\
\hline
\end{tabular}

\subsection{In solar current-carrying plasma loops}

From Sect. 2, we show that there are a lot of similarities between tokamaks and solar current-carrying plasma loops. Both of them are current-carrying plasma loops, all have localized magnetic mirror configurations, all charged particles may be classified into two groups (trapped and passing particles), all have plasma pressure gradient, and in some of the solar flare loops, the plasma is situated in the banana regime. Thus, it is reasonable to suppose that bootstrap currents possibly occur in solar current-carrying plasma loops. We suppose that the total current of solar plasma loops is composed of two parts: convectional current $j_{\mathrm{c}}$ and neoclassical bootstrap current $j_{\mathrm{b}}$. With the magnetic mirror ratio $\zeta(x)$, we write the critical incidence angle $\theta_{\mathrm{c}}$ and the paralleled velocity of passing electrons as $\sin \theta_{\mathrm{c}}=\sqrt{1 / \zeta(x)}=f^{1 / 2}$ and $v_{\mathrm{po}}=v_{\mathrm{th}} \cos \theta_{\mathrm{c}}(1-f)^{1 / 2}$, respectively.

Then the fraction of trapped electrons is $(1-f)^{1 / 2}$. The expression of bootstrap current density in solar current-carrying plasma loops is written in comparison with Eq. (20) as

$j_{\mathrm{bs}}(r)=-\frac{2 c(1-f)^{1 / 2}}{B_{\theta}} T_{\mathrm{e}} \frac{\mathrm{d} n_{\mathrm{e}}}{\mathrm{d} r}$.

The appearance of bootstrap current will disturb the equilibrium of the magnetic tube and result in a redistribution of pressure. As the bootstrap current is coupled strongly with the distribution of total current, we may suppose that the distribution of total current is approximately dominated by the pinch effect, with expressions in Eqs. (6) and (7). We adopt Eqs. (14), (15) as the equilibrium distribution of the plasma pressure and Eqs. (8), (9) as the equilibrium distribution of the poloidal magnetic field, substitute Eqs. (15), (9), and the relation $p \simeq 2 n_{\mathrm{e}} T_{r}$ into Eq. (21), we rewrite the expression of bootstrap current density in solar current-carrying plasma loops:

$j_{\mathrm{bs}}(r)=\frac{(1-f)^{1 / 2} I}{\pi a^{2}}\left(\frac{1}{1+x^{2}}\right)^{2}$.

The bootstrap current is $I_{\mathrm{bs}}=\int j_{\mathrm{bs}}(r) 2 \pi r \mathrm{~d} r$. From Eqs. (6) or (7), we obtain the fraction of bootstrap current in the total current:

$f_{\mathrm{bs}}=\frac{j_{\mathrm{bs}}(r)}{j(r)}=\frac{(1-f)^{1 / 2}}{2}$.

Equations (22) and (23) vary with the extrapolated model of magnetic field, i.e. the function of $f$. Here we quote a extrapolated expression as follows (Takakura 1972; Huang et al. 1999):

$f=(1+h / d)^{-3}$.

Then the fraction of bootstrap current is about:

$f_{\mathrm{bs}}=\frac{1}{2}\left[1-(1+h / d)^{-3}\right]^{1 / 2}$.

The bootstrap current will induce a magnetic field in the same way as the conductive current, and of course it satisfies 
Table 2. The loop's parameters (from Bray et al. 1991) and the bootstrap current calculating results.

\begin{tabular}{lccc}
\hline \hline Parameter & Cool loop & Hot loop & Flare loop \\
\hline$R, L(\mathrm{~m})$ & $1.0 \times 10^{7}$ & $3.0 \times 10^{7}$ & $7.0 \times 10^{6}$ \\
$a_{0},(\mathrm{~m})$ & $1.0 \times 10^{6}$ & $5.0 \times 10^{6}$ & $2.8 \times 10^{6}$ \\
$d,(\mathrm{~m})$ & $7.85 \times 10^{7}$ & $4.0 \times 10^{8}$ & $1.0 \times 10^{7}$ \\
$T_{\mathrm{e}},(\mathrm{eV})$ & 10.0 & 300.0 & 900.0 \\
$B_{\varphi 0},(\mathrm{~T})$ & 0.1 & 0.1 & 0.1 \\
$n_{\mathrm{e}},\left(\mathrm{m}^{-3}\right)$ & $1.0 \times 10^{16}$ & $2.0 \times 10^{15}$ & $1.3 \times 10^{15}$ \\
$g$ & $1.2 \times 10^{-5}$ & $1.8 \times 10^{-2}$ & 1.05 \\
$I,(\mathrm{~A})$ & $4.0 \times 10^{10}$ & $1.0 \times 10^{11}$ & $1.0 \times 10^{11}$ \\
$I_{\mathrm{bs}},(\mathrm{A})$ & - & - & $4.46 \times 10^{10}$ \\
$f_{\mathrm{bs}},(\%)$ & - & - & 44.6 \\
\hline
\end{tabular}

Ampère's Law. The occurrence of bootstrap current changes both the current density profile and the poloidal magnetic field distribution, and affects the evolution of magnetic reconnection and the tearing mode instability in plasma loops. When the bootstrap current $f_{\mathrm{b}}$ becomes large enough, a new kind of MHD instability, the neoclassical tearing mode (NTM), will be triggered. The growth rate is obtained first by Qu \& Callen (1985), and modified by Tan (2003):

$\gamma_{\mathrm{NTM}}=6.61\left(\tau_{\mathrm{A}}\right)^{-1} \triangle^{\prime-2 / 3}\left(j_{\mathrm{b}} \alpha \dot{B}_{\theta}\right)^{2 / 3} S^{-1 / 3}$.

Its reciprocal indicates the growth time $\tau_{\mathrm{NTM}}=0.1513 \times$ $\left(\frac{\Delta^{\prime}}{j_{\mathrm{bt}} \alpha \dot{B}_{\theta}}\right)^{2 / 3} \tau_{\mathrm{A}}^{2 / 3} \tau_{\mathrm{r}}^{1 / 3}$, here $S$ is the magnetic Reynolds number, $S=\tau_{\mathrm{r}} / \tau_{\mathrm{A}}, \Delta^{\prime}$ is the tearing mode instability factor, $\alpha$ the mode number, $\tau_{\mathrm{A}}$ the Alfvén time scale, and $\tau_{\mathrm{r}}$ the resistive diffusive time. The first observation of NTM is obtained by Chang et al. (1995). As a contrast, the growth rate of classical tearing mode (CTM) is derived by Furth et al. $(1963,1973)$ as:

$\gamma_{\mathrm{CTM}}=0.755\left(\tau_{\mathrm{A}}\right)^{-1}{\Delta^{\prime / 5}}^{4 / 2}\left(\dot{B}_{\theta}\right)^{2 / 5} S^{-3 / 5}$,

and its growth time is $\tau_{\mathrm{CTM}}=1.325\left(\triangle^{\prime 2} \alpha \dot{B_{\theta}}\right)^{-2 / 5} \tau_{\mathrm{A}}^{2 / 5} \tau_{\mathrm{r}}^{3 / 5}$. For solar plasma $S \sim 10^{9}-10^{15}$, if the bootstrap current occurs in solar plasma loop and trigger NTM, then $\gamma_{\mathrm{NTM}} \sim 10^{3}-10^{5} \gamma_{\mathrm{CTM}}$. This shows that the growth rate of NTM is much higher than that of CTM, which may cause much fast eruptive events, and the plasma loops may become even more unstable. Using this mechanism, we can explain some features of solar eruptive events.

\section{Bootstrap current in a solar flare event}

We may roughly classify the typical solar plasma loops into two groups: hot loops and cold loops. The first and second columns in Table 2 are the parameters in typical coronal loops (Bray et al. 1991) and calculating results. As an approximation, we let $R \sim L \sim 2 h$. The typical values of total current are estimated from the combination of the model of Zaitsev et al. (1998) and the observations of vector magnetography (Khodachenko et al. 2003).

From Table 2, we find that bootstrap current is negligible in typical solar plasma loops. With average parameters of the typical solar plasma loops in Table 2 , the factor $g \ll 1.0$ and the plasma beyond the banana regime, so it is impossible to get a meaningful value for the bootstrap current.

However, the parameters of solar plasma loops vary in a broad range. For example, plasma density $n_{\mathrm{e}}$ may change from $10^{14} \mathrm{~m}^{-3}$ to $10^{17} \mathrm{~m}^{-3}$, and temperature $T_{\mathrm{e}}$ change from $10^{5} \mathrm{~K}$ to several $10^{7} \mathrm{~K}$, loop radius $R, L$ from $5000 \mathrm{~km}$ to $50000 \mathrm{~km}$, etc. The range of the $\lambda_{\mathrm{c}}$ magnitude is about $1.0 \times 10^{2}-1.0 \times 10^{8} \mathrm{~m}$,
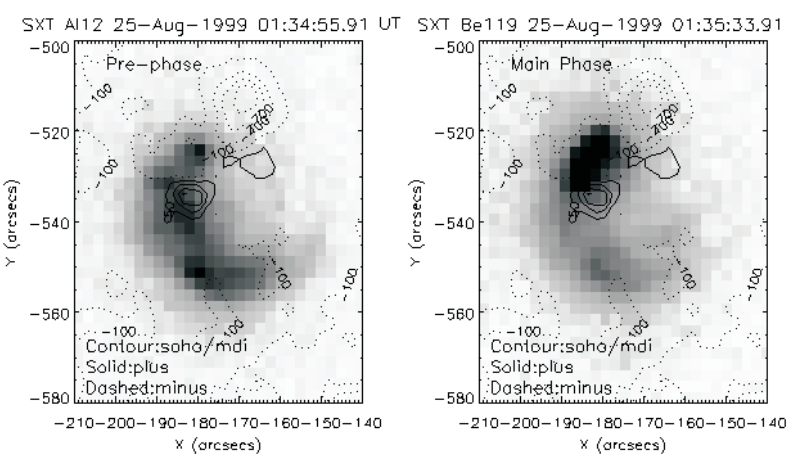

Fig. 3. An M 3.6 flare loop of YOHKOH/SXT overlaid by SOHO/MDI on 25 Aug. 1999.

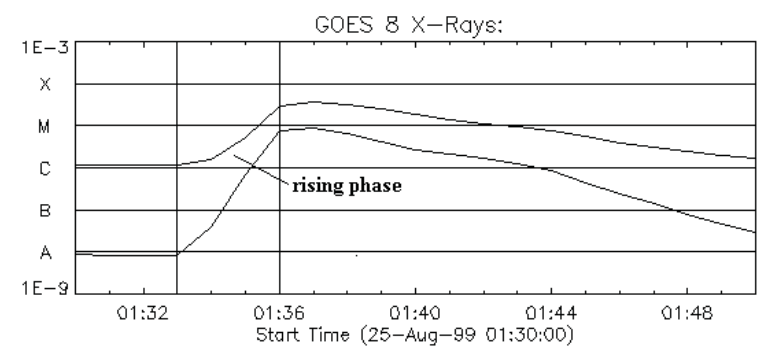

Fig. 4. The temporal profile of GOES X-rays of the loop flare on 25 Aug. 1999.

and the factor $g$ is about $10^{-5}-20$. If the plasma at $T_{\mathrm{e}}=1 \mathrm{keV}$, $n_{\mathrm{e}}=10^{15} \mathrm{~m}^{-3}$, we find $g>1$ when $L \leq 1.2 \times 10^{7} \mathrm{~m}$. Likewise, some of solar loops satisfy the conditions of the banana regime. In this case, it is reasonable to suppose that the neoclassical effect is measurable in these loops. A flare event with a plasma loop is selected in comparison with the conditions of the bootstrap current as follows.

The third column in Table 2 shows an M 3.6 GOES flare event on 25 Aug. 1999 from 01:32 UT to 01:40 UT, with the maximum at 01:36 UT in AR8674 (Huang et al. 2003). Figure 3 is the flare loop of $\mathrm{YOHKOH/SXT} \mathrm{overlaid} \mathrm{by} \mathrm{the} \mathrm{magnetic} \mathrm{map}$ of SOHO/MDI, in which we can estimate the geometrical parameters of the loop in Table 2. The loop radius $R$ and section radius $a$ are measured by the contour with $50 \%$ of the maximum intensity in the image of $\mathrm{YOHKOH} / \mathrm{SXT}$.

From the third column of Table 2, we find that the fraction of bootstrap current is about $f_{\mathrm{b}}=44.6 \%$, and the total bootstrap current is about $4.46 \times 10^{10} \mathrm{~A}$ in the above flare loop, and this value is even larger than that of many tokamaks (Table 1). As the neoclassical effect is proportional to $f_{\mathrm{b}}$, it will be very obvious in the above loop.

Moreover, NTM may occur in the above loop, in which the magnetic Reynolds number is $S=2.349 \times 10^{15}$, and the growing timescale of NTM is $t_{\mathrm{NTM}}=9.7 \times 10^{2}(\mathrm{~s})$, while $t_{\mathrm{CTM}}=1.06 \times$ $10^{8}(\mathrm{~s})$, so, $t_{\mathrm{NTM}} \ll t_{\mathrm{CTM}}$. Figure 4 shows the X-ray temporal profile of GOES 8, which reflects the thermal emission feature in the flare. The rising phase in this profile is about $3 \mathrm{~min}$. Figures 5 and 7 are the time profiles, respectively, of radio emissions (NoRP) and hard X-rays (Yohkoh/HXT), which reflect the features of non-thermal emissions, with the thermal features at lower frequencies and energies. The duration of the impulsive phase is about minute, so, the NTM growing timescale fits the observations much better than those of CTM.

In fact, the flare loop in this event looks like a single loop. With Figs. 4 and 7, the temporal profiles are composed of a prephase and a main phase. The main phase occurs 3 min later 


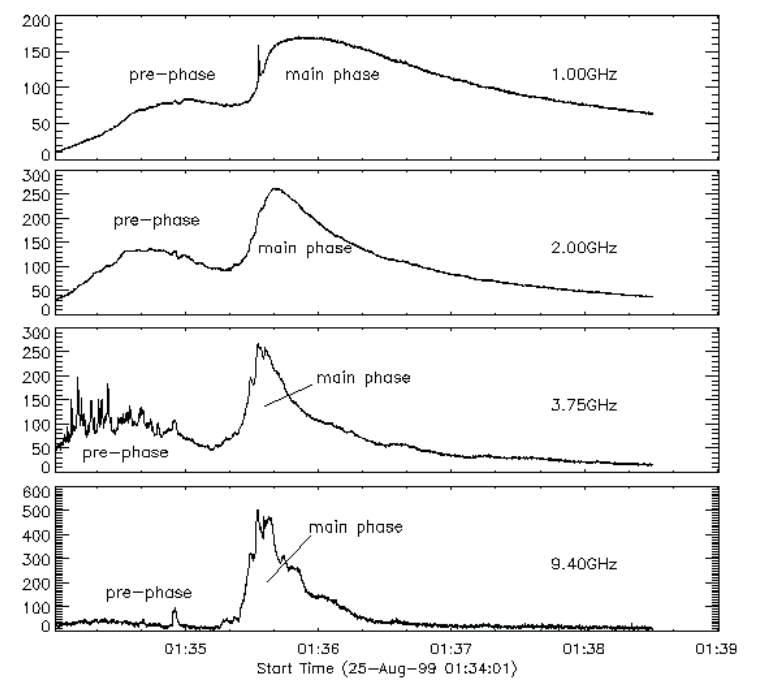

Fig. 5. Temporal profile of radio emission (NoRP) on 25 Aug. 1999.
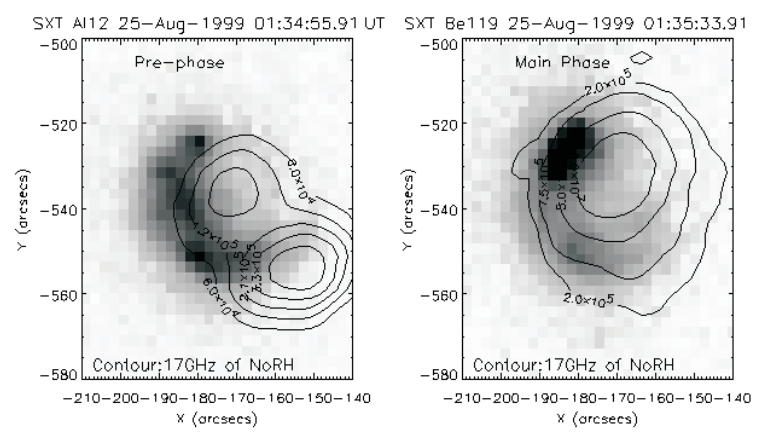

Fig. 6. The contour of $17 \mathrm{GHz}(\mathrm{NoRH})$ overlaid on Yohkoh/SXT of the flare event on 25 Aug. 1999.
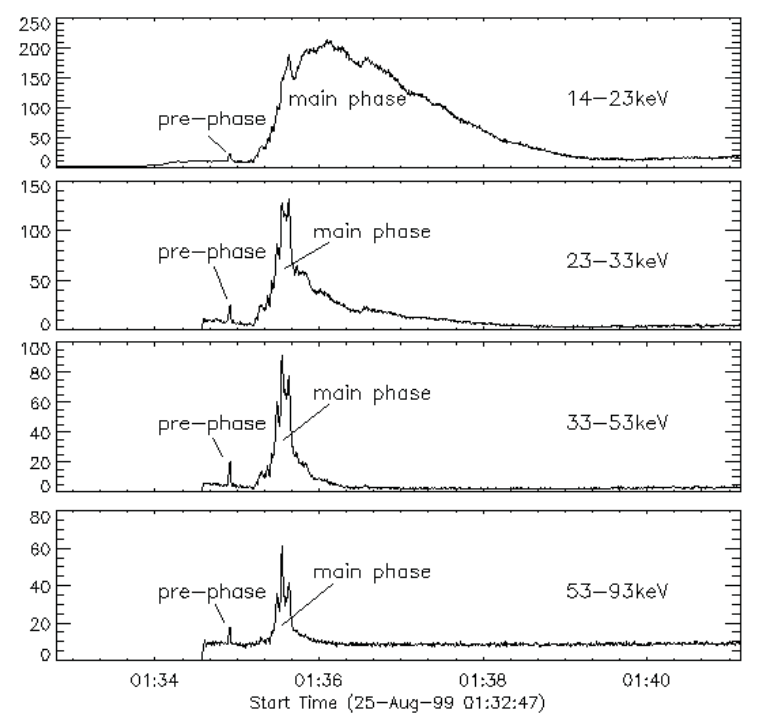

Fig. 7. Temporal profile of Yohkoh/HXT on 25 Aug. 1999.

than the pre-phase. The total duration of the pre-phase and main phase is about 3 min (from 01:33 to 01:36), which is comparable to the duration of the rising phase in Fig. 4, as well as to the growing time of NTM. From the left panels of Figs. 3, 6, and 8, the pre-phase takes place in a large area within the loop and near the loop top, and the main phase takes place near one foot-point from the right panels of Figs. 3, 6, and 8. These results may
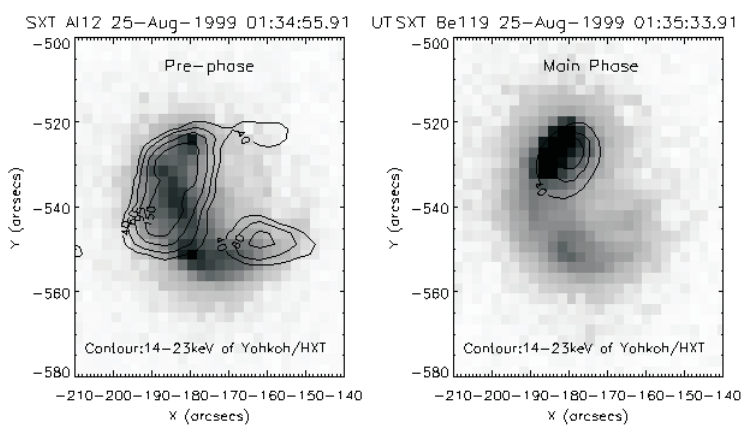

Fig. 8. The contour of Yohkoh/HXT overlaid on Yohkoh/SXT for the flare event on 25 Aug. 1999.

support the existence of bootstrap current, the NTM instability, and magnetic reconnection may take place inside the loop, not only in the area above the loop top, which is a hint that the bootstrap current and NTM may provide a new mechanism for the plasma heating and particle acceleration.

\section{Conclusions and discussions}

Based on the similarities in magnetic configurations and plasma behaviors between tokamaks and solar current-carrying plasma loops, we suggest that the neoclassical effect does work in some of the solar plasma loops. In typical coronal loops, the neoclassical effect is too weak to be considered, while in some flare loops with high temperature, the neoclassical effect is very strong and the bootstrap current really exists. For example, in the flare loop that occurred during the event on 25 Aug. 1999, the fraction of bootstrap current in total current $\left(f_{\mathrm{bs}}\right)$ may reach $44.6 \%$.

From derivations in Sect. 3, we can know that the fraction of banana particles (trapped particles) relative to the total amount of particles is an important parameter for the bootstrap current. In tokamaks the fraction of trapped particles is about $\epsilon^{1 / 2}$, while in solar current-carrying plasma loops $(1-f)^{1 / 2}$. Based on the parameters from the book by Bray et al. (1991), the fraction is about $25-55 \%$ and $30-50 \%$ in typical cool and hot coronal loops respectively, while in the solar flare loop, the fraction is about $50-90 \%$. As a comparison, in typical modern tokamaks, the inverse aspect ratio $\epsilon \sim 0.3-0.6$, the fraction of trapped particles is about $55-75 \%$.

Because of the low values of plasma beta in the solar corona, the force-free magnetic field is widely accepted in solar coronal loops. Based on the data from Bray et al. (1991) and the expression from the book by Priest \& Forbes (2000), the beta value in solar flare loops is about $\beta \sim 0.01-3.0 \%$, while in tokamaks, the maximum value is about $8 \%$ for the case of circular magnetic surface, and the typical value is about $2-4 \%$ (Boozer 2004 ). In both of the above cases, $\beta \ll 1$, all of them are low beta plasma. We may regard them as a force-free field, approximately. In the derivation of the bootstrap current, the neoclassical tearing modes, and other instabilities of the circular magnetic surface, we may use the assumption of force-free field, i.e., low beta and $\boldsymbol{j} \times \boldsymbol{B} \sim 0$ (Bickerton et al. 1971; Qu \& Callen 1985; Peeters et al. 2000). Thus, some properties of the solar flare loops are similar to tokamaks with a circular magnetic surface.

However, in some modern tokamaks, magnetic surfaces are always non-circular and the beta value may exceed $10 \%$. In such cases, it isn't suitable to deal with the derivation of bootstrap current under the assumption of a force-free field. The bootstrap current may occur in the plasma torus not only with the forcefree field, but also with the non-force-free field. And the high 
beta plasma is one of the intended targets in magnetic confinement fusion (MCF). In fact, when we analyze the current penetration, edged localized modes, and the nonlinear evolution of the plasma instabilities in modern tokamaks, the localized beta value may exceed $10 \%$ where we should consider the non-forcefree field. In this work, we simply use a simplified way to study the possibility of a bootstrap current in the solar plasma loops, but will make a further effort in our forthcoming work.

The bootstrap current is a new possible complement to the current model in solar magnetic current-carrying plasma loops. With such a consideration of the new current profile, we may investigate the evolution of tearing mode instability and the magnetic reconnection in the loops. The occurrence of bootstrap current changes the profile of current density and poloidal magnetic field. When it becomes large enough, the neoclassical tearing mode will be triggered in the whole loop to cause a fast magnetic reconnection, which may provide an effective mechanism of the solar plasma heating and particle acceleration.

Electric currents in magnetic plasma loops governs the total amount of energy stored in the loops and released in the process of magnetic reconnection. In the meantime, the tearing modes may result in the plasma current redistribution and lead to the formation of magnetic islands, while plasma loops may change into filaments along the magnetic field lines. With this analysis, we can explain the observations of the low filling factors of hot coronal plasma loops (Martens et al. 1985).

In our forthcoming work, we will try to apply the concept of the disruptive instability in tokamaks to solar current-carrying plasma loops as a new model for explaining the process of the solar and stellar eruptive events. It is emphasized that the magnetic reconnection triggered by the tearing modes takes place in the whole loop, and is not only localized in the current sheet above the loop top. And this will be more effective for the plasma heating and particle acceleration. With the high-resolution observations of RHESSI and other instruments, we may be able to distinguish the fine structure of loops in plasma temperature, density, magnetic field, and their distribution to better understand the solar eruptive events.

Acknowledgements. The authors would like to thank the anonymous referee very much for the helpful and significant comments on our paper. We are grateful for the useful discussions with Prof. Wang D. Y., Dr. Ji H. S., and Dr. Wu
D. J. at the Purple Mountain Observatory, and with Prof. Dong J. Q. at the Southwestern Institute of Physics, Chengdu, China. This study is supported by the NFSC projects No. 10333030 and 10273025, and "973" program with No. G2000078403.

\section{References}

Alfvén H., \& Carlqvist, P. 1967, Sol. Phys., 1, 220

Ashbourn, J. M. A., \& Woods, L. C. 2004, ApJ, 602, 1021

Bennett, W. H. 1934, Phys. Rev., 45, 90

Bickerton, R. J., Connor, J. W., \& Taylor, J. B. 1971, Nature Phys. Sci., 229, 110 Boozer, A. H. 2004, Rev. Mod. Phys., 76, 1071

Bray, R. J., Cram, L. E., Durrant, C. J., \& Loughhead, R. E. 1991, Plasma Loops in the Solar Coroma (New York: Cambridge University Press)

Canfield, R. C., De La Beaujardiere, J. F., Fan, Y., et al. 1993, ApJ, 411, 362

Chang, Z., Callen, J. D., \& Fridrickson, E. D. 1995, Phys. Rev. Let., 74, 4663

Coenelis, D. J. 1988, Astrophys. Space Sci., 144, 319

Cordey, J. G., Challis, C. D., \& Stubberfield, P. M. 1988, Plasma phys. Control. Fusion, 30, 1625

Furth, H. P., Killeen, J., \& Rosenbluth, M. N. 1963, Phys. Fluids, 6, 459

Furth, H. P., Rutherford, P. H., \& Selberg, H. 1973, Phys. Fluids, 16, 1054

Huang, G. L., Fu, Q. J., \& Qin, Z. H. 1999, Astrophys. Space Sci., 266, 389

Huang, G. L., Wu, H. A., Grechnev, V. V., Sych, R. A., \& Altyntsev, A. T. 2003, Sol. Phys., 213, 341

Hirshman, S. P. 1988, Phys. Fluids, 31, 3150

Khodachenko, M., Haerendel, G., \& Rucker, H. O. 2003, A\&A, 401, 721

Kikuchi, M., \& Azumi, M. 1995, Plasma Phys. Control. Fusion, 37, 1215

Martens, P. C. H., Van Den Oord, G. H. J., \& Hoyng, P. 1985, Sol. Phys., 96, 253

Peeters, A. G. 2000, Plasma Phys. Control. Fusion, 42, B231

Petty, C. C., Politzer, P. A., \& Lin-Liu, Y. R. 2005, Plasma Phys. Control. Fusion, 47, 1077

Priest, E., \& Forbes, T. 2000, Magnetic Reconnection-MHD Theory and Applications (Cambridge: Cambridge University Press)

Qu, W. X., \& Callen, J. D. 1985, UWPR-85-5, University of Wisconsin, Wisconsin

Schuller, F. C. 1995, Plasma phys. Control. Fusion, A135, 37

Sen, H. K., \& White, M. L. 1972, Sol. Phys., 23, 146

Severny, A. B. 1964, Space Sci. Rev., 3, 451

Spadaro, D. 2003, Mem. S. A. It. Suppl., 3, 30

Strong, K. T., \& the Yohkoh team 2003, Space Sci. Rev., 70, 133

Takatura, T. 1972, Sol. Phys., 77, 151

Tan, B. L. 2003, MA thesis (in Chinese), Southwestern Institute of Physics, Chengdu, China

Tanaka, K., Watanabe, T., \& Nishi, K. 1982, ApJ, 254, L59

Wesson, J. 1997, Tokamak, 2nd ed. (Oxford: Claarendon Press)

Zaitsev, V. V., \& Stepanov, A. V. 1992, Sol. Phys., 139, 343

Zaitsev, V. V., Stepanov, A. V., Urpo, S., \& Pohjolainen, S. 1998, A\&A, 337, 887

Zarnstorff, M. C., \& Prager, S. C. 1986, Phys. Fluids, 29, 308 